\title{
A NOTE ON THE COHEN-MACAULAY TYPE OF LINES IN UNIFORM POSITION IN $A^{n+1}$
}

\author{
WILLIAM C. BROWN
}

\begin{abstract}
Let $E_{1}^{\prime} \ldots \ldots \mathscr{L}^{\prime}$, be $s$-distinct lines in $\mathbf{A}_{h}^{\prime \prime+1}$ passing through the origin. Assume $s=\left({ }_{n}+d\right)-\lambda$ where $n, d \geqslant 2$. If $E_{1}, \ldots, E_{\text {, }}$ are in generic $s$-position, and $\lambda=0,1, \ldots, n-1$, then the Cohen-Macaulay type, $t\left(E_{1}, \ldots, E_{1}\right)$. of $E_{1} \ldots \ldots E_{1}$, is given by the following formula: $t\left(E_{1}, \ldots, E_{1}\right)=\left(n_{n-1}^{n+d}\right)-\lambda$. This formula is known to be false for $\lambda=n$. In this paper, we show that if $L_{1} \ldots \ldots . \dot{L}$, are in uniform position, and $\lambda=n$, then $t\left(E_{1}, \ldots, E_{s}\right)=\left(\begin{array}{c}n+d \\ n-1\end{array}\right)-n$.
\end{abstract}

Introduction. Throughout this paper, $k$ will denote an algebraically closed field of arbitrary characteristic. We shall let $\mathbf{A}_{k}^{n+1}$ and $\mathbf{P}_{k}^{n}$ denote affine $(n+1)$-space and projective $n$-space over $k$ respectively.

If $E_{1}, \ldots, E_{s}$ are $s$-distinct lines in $\mathbf{A}_{k}^{n+1}$ passing through the origin, we shall identify $L_{i}$, with its direction numbers $P_{i} \in P_{k}^{n}, i=1, \ldots, s$. Let $s=k\left[X_{0}, \ldots, X_{n}\right]$ denote the coordinate ring of $\mathbf{A}_{k}^{n+1}$ (or $\mathbf{P}_{k}^{n}$ ). The $j$ th homogeneous piece of $s$ will be denoted by $\varsigma_{j}$. Thus, $\varsigma_{j}$ is a $k$-vector space of dimension $\left(\begin{array}{c}n+j \\ n\end{array}\right)$. Set $\nu(j)=\left(\begin{array}{c}n+j \\ n\end{array}\right)$. Let $\left\{g_{1}, \ldots, g_{\nu(j),}\right\}$ be a vector space basis of $S_{,}$. We can then form an $[s \times \nu(j)]$-matrix $G_{j}\left(E_{1}, \ldots, L_{s}\right)$ as follows: the $(\alpha, \beta)$ th entry of $G_{j}\left(L_{1}, \ldots, L_{s}\right)$ is $g_{\beta j}\left(P_{\alpha}\right)$. We shall denote the rank of the matrix $G_{j}\left(E_{1}, \ldots, E_{s}\right)$ by $\operatorname{rk}\left(G_{j}\left(E_{1}, \ldots, E_{s}\right)\right)$.

The lines $E_{1}, \ldots, E_{s}$ are said to be in generic s-position if $\operatorname{rk}\left(G_{j}\left(E_{1}, \ldots E_{s}\right)\right)=$ $\min \{s, \nu(j)\}$ for all $j \geqslant 1$. If $0<t \leqslant s$, we say $E_{1} \ldots \ldots, E_{s}$ are in generic $t$-position if every $t$-subset of $\left\{E_{1}, \ldots, E_{s}\right\}$ is in generic $t$-position. Finally, the set of lines $\mathfrak{E}_{1}, \ldots, \mathfrak{E}_{s}$ is said to be in uniform position if it is in generic $t$-position for every $0<t \leqslant s$

Now let $E_{1}, \ldots, E_{s}$ be in generic $s$-position in $\mathbf{A}_{k}^{n+1}$. We assume $s=\nu(d)-\lambda$. Here $0 \leqslant \lambda \leqslant \nu(d-1)+1$. To avoid trivial cases, we shall assume throughout the rest of this paper that $n \geqslant 2$, and $d \geqslant 2$. By the Cohen-Macaulay type, $t\left(E_{1}, \ldots E_{s}\right)$, of $e_{1}, \ldots, e_{s}$, we shall mean the Cohen-Macaulay type of the corresponding local ring (at the origin) of the reduced curve $\cup_{i=1}^{s} E_{i}$. The reader is referred to [2] for all pertinent definitions not given here.

$t\left(\mathfrak{L}_{1}, \ldots, \mathfrak{E}_{s}\right)$ is known when $\lambda=0,1, \ldots, n-1$. A precise formula is given as follows:

$$
t\left(\varrho_{1}, \ldots, \varrho_{s}\right)=\left(\begin{array}{c}
n+d-1 \\
n-1
\end{array}\right)-\lambda, \quad \lambda=0,1, \ldots, n-1
$$

Received by the editors August 11, 1982 and, in revised form. September 3, 1982. 1980 Mathematics Subject Classification. Primary 13H10, 14H20, 13 HI5.

Key words and phrases. Cohen-Macaulay type, generic s-position, uniform position. 
When $\lambda=0$, equation (1) follows from Proposition 16 in [3]. For $\lambda=1,2, \ldots, n-1$, equation (1) was established by L. Roberts in [5].

When $\lambda=n$, Roberts showed [5] that equation (1) continues to hold "generically". More precisely, let us topologize the lines through the origin in $\mathbf{A}_{k}^{n+1}$ by identifying $E_{1}, \ldots, E_{s}$ with the point $\left(P_{1}, \ldots, P_{s}\right)$ in $s$-copies $\mathbf{P}_{k}^{n} \times \cdots \times \mathbf{P}_{k}^{n}$ of $\mathbf{P}_{k}^{n}$. Then, when $\lambda=n$, Roberts showed that there exists a nonempty, Zariski open subset $U$ of $\mathbf{P}_{k}^{n} \times \cdots \times \mathbf{P}_{k}^{n}(s=(\nu(d)-n)$-copies here $)$ such that $t\left(\varrho_{1}, \ldots, \varrho_{s}\right)=\left(\begin{array}{c}n+d-1 \\ n-1\end{array}\right)-n$ whenever $\left(\varrho_{1}, \ldots, \ell_{s}\right) \in U$.

The exact nature of the open set $U$ is not clear. For instance, $U^{\prime}=\left\{\left(Q_{1}, \ldots, Q_{1}\right) \in\right.$ $\mathbf{P}_{k}^{n} \times \cdots \times \mathbf{P}_{k}^{n} \mid \mathscr{L}_{1}, \ldots, \mathscr{L}_{s}$ are in generic $s$-position in $\left.\mathbf{A}_{k}^{n+1}\right\}$ is easily seen to be a nonempty, open subset of $\mathbf{P}_{k}^{n} \times \cdots \times \mathbf{P}_{k}^{n}$. But, for $\lambda=n$, equation (1) is not true on $U^{\prime}$. Concrete examples can be found in [1]. In this paper, we give a specific open set upon which equation (1) continues to hold when $\lambda=n$. Namely, let $U^{\prime \prime}=$ $\left\{\left(\varrho_{1}, \ldots, \varrho_{s}\right) \in \mathbf{P}_{k}^{n} \times \cdots \times \mathbf{P}_{k}^{n} \mid \varrho_{1}, \ldots, \varrho_{s}\right.$ are in uniform position $\}$. One easily checks that $U^{\prime \prime}$ is a nonempty, open subset of $\mathbf{P}_{k}^{n} \times \cdots \times \mathbf{P}_{k}^{n}$ contained in $U^{\prime}$. We shall show that the Cohen-Macaulay type of $L_{1}, \ldots, L_{s}$ is given by equation (1) when $\lambda=n$, and $\left(\varrho_{1}, \ldots, \varrho_{s}\right) \in U^{\prime \prime}$.

The question of the "generic" Cohen-Macaulay type of lines in $\mathbf{A}_{k}^{3}$ (i.e. $n=2$ ) has been completely solved in [4]. The results there seem to indicate that "uniform position" is not a strong enough hypothesis to give an equation like (1) when $n>2$, and $\lambda>n$.

Finally, the author assumes the reader is familiar with the contents of [ 2,3 and 4$]$ and will use results from these three papers freely.

\section{Main result.}

THEOREM. Let $\mathcal{L}_{1}, \ldots, \mathcal{L}_{s}$ be $s$-distinct lines passing through the origin in $\mathbf{A}_{k}^{n+1}$. We assume $s=\left(\begin{array}{c}n+d \\ n\end{array}\right)-n$ with $n, d \geqslant 2$. If $\varrho_{1}, \ldots, \varrho_{s}$ are in uniform position, then

$$
t\left(\varrho_{1}, \ldots, e_{s}\right)=\left(\begin{array}{c}
n+d-1 \\
n-1
\end{array}\right)-n .
$$

Proof. Recall $\delta=k\left[X_{0}, \ldots, X_{n}\right]$. Let $g$ denote the ideal of $\cup_{i=1}^{s} \mathcal{L}_{i}$ in $\S$. Thus, $g$ is a homogeneous, unmixed, radical ideal of height $n$ in $\mathcal{\delta}$. We first argue that we can assume without loss of generality that every nonzero form in $g_{d}$ is irreducible. Here, $q_{d}$ denotes the $d$ th homogeneous piece of 9 .

We first consider the special case $d=2$. Then $s=\left(\begin{array}{c}n+2 \\ n\end{array}\right)-n$ with $n \geqslant 2$. Clearly, $2=\min \left\{j \mid\left(\begin{array}{c}n+j \\ j\end{array}\right)>s\right\}$ and, thus, it follows from [2, Remark (3), p. 8] that $g_{2} \neq(0)$. Suppose $q_{2}$ contains a nonzero, reducible quadratic form $F$. Then $F=l_{1} l_{2}$ with $l_{i}$ a one form in $\delta$. $\left\{P_{1}, \ldots, P_{s}\right\} \subseteq V(F)=V\left(l_{1}\right) \cup V\left(l_{2}\right)$ in $P_{k}^{n}$. Suppose $\left\{P_{1}, \ldots, P_{s}\right\} \subseteq$ $V\left(l_{1}\right)$. Again using [2, Remark (3), p. 8], we would have $\nu(1)-1 \geqslant s$. This last inequality implies $4 n \geqslant(n+2)(n+1)$ which is impossible since $n \geqslant 2$. Thus, some of the $P_{i}$ lie on $l_{1}$ and some on $l_{2}$. Let $t_{i}$ denote the number of points in $\left\{P_{1}, \ldots, P_{s}\right\} \cap V\left(l_{i}\right), i=1,2$. Then $t_{i} \geqslant 1$, and $t_{1}+t_{2} \geqslant s$. Again applying [2, Remark (3), p. 8], we have the following inequality:

$$
2[\nu(1)-1] \geqslant t_{1}+t_{2} \geqslant s .
$$


The inequality in (3) implies $6 n \geqslant n^{2}+3 n+2$. This is possible only in the case $n=2$. But then $s=4$, and we have 4-lines in uniform position in $\mathbf{A}_{k}^{3}$. Now it is well known (e.g. [2, Theorem 7]) that 4-lines in uniform position in $\mathbf{A}_{k}^{3}$ have a Gorenstein singularity at the origin. Therefore, $t\left(\varrho_{1}, \varrho_{2}, \varrho_{3}, \varrho_{4}\right)=1=\left(\begin{array}{c}2+2-1 \\ 2-1\end{array}\right)-2$, and our theorem is correct in this case. So, when $d=2$, we can assume $n \geqslant 3$. In this case, the above argument shows any $F \in \Phi_{2}-(0)$ is irreducible.

Now assume $d \geqslant 3$. We first note that $\nu(d-1)<s<\nu(d)$ (these inequalities hold for $d=2$ as well). In particular, $d=\min \{j \mid \nu(j)>s\}$. Thus, $\Phi_{j}=0$ for $j<d$, and $q_{d} \neq 0$. Since $d \geqslant 3$, we have $\nu(d-1)+n \leqslant s$. Thus, if we write $s=\nu(d-1)$ $+h$, then $n \leqslant h<\left(\begin{array}{c}n+d-1 \\ n-1\end{array}\right)$. It now follows immediately from [4, Theorem 3.4] that every $F \in G_{d}-(0)$ is irreducible. This completes all cases for $d$, and, so, henceforth, we shall assume that any nonzero form in $\mathscr{G}_{d}$ is irreducible.

Since $\mathcal{L}_{1}, \ldots, \mathcal{L}_{s}$ are in generic $s$-position, $\operatorname{dim}_{k}\left\{g_{j}\right\}=\nu(j)-\min \{s, \nu(j)\}$ for $j=1,2 \ldots$ In particular, $\operatorname{dim} \Phi_{d}=n$. Thus, $\Phi_{d}=\bigoplus_{i=1}^{n} k F_{i}$ with $F_{1}, \ldots, F_{n}$ irreducible, $d$-forms in 9 .

Let the associated primes of $q$ be denoted by $\mathscr{P}_{1}, \ldots, P_{s}$, and let the associated primes of the ideal $\left(F_{i}, F_{j}\right)$ be denoted by $Q_{1}^{i, j}, \ldots, Q_{r(i, j)}^{i, j}$. Here $1 \leqslant i \neq j \leqslant n$, and $r(i, j)$ is some positive integer. Since $\delta$ is a Cohen-Macaulay ring, the grade of 4 is $n$. Since $\left\{F_{i}, F_{j}\right\}$ are linearly independent, irreducible forms, the grade of $\left(F_{i}, F_{j}\right)$ is two. In particular, the maximal ideal $\left(X_{0}, \ldots, X_{n}\right)$ properly contains any $\mathscr{P}_{i}$ or $Q_{t}^{i . j}$. Thus, if we set

$$
W=\left\{\bigcup_{i=1}^{s} \mathscr{P}_{i} \cap \mathcal{\delta}_{1}\right\} \cup\left\{\bigcup_{i \neq j}\left(\bigcup_{t} Q_{i}^{i, j} \cap \S_{1}\right)\right\},
$$

then $\delta_{1}-W$ is nonempty. Let $g \in \mathcal{S}_{1}-W$. Then $g$ is not in any associated prime of 9 or of $\left(F_{i}, F_{j}\right)$ for $i \neq j$. Changing coordinates in $\mathbf{P}_{k}^{n}$ if need be, we can assume $g=X_{0}$ without any loss in generality.

Now set $\Re=\delta / 9$, the coordinate ring of $\cup_{i=1}^{s} R_{i}$. Let $x_{0}$ denote the image of $X_{0}$ in $R$. Then $x_{0}$ is a nonzero-divisor in $R$. Also, for any $1 \leqslant i \neq j \leqslant n,\left\{F_{1}, F_{j}, X_{0}\right\}$ is a regular sequence of length three in $\mathcal{S}$. Since $F_{i}, F_{j}$ and $X_{0}$ are all forms, $\left\{X_{0}, F_{i}, F_{j}\right\}$ is also a regular sequence of length three in $\mathcal{S}$.

Set $\mathcal{T}=k\left[Y_{1}, \ldots, Y_{n}\right]$. Here $Y_{1}, \ldots, Y_{n}$ are indeterminates over $k$. Let $\psi: \delta \rightarrow \sigma$ be the $k$-algebra epimorphism given by $\psi\left(X_{0}\right)=0, \psi\left(X_{i}\right)=Y_{i}$ for $i=1, \ldots, n$. Then we have a natural $k$-algebra epimorphism $\sigma: \mathcal{T} \rightarrow \Re / x_{0} \Re$ such that the kernel of $\sigma$ is $\psi(9)$, and $\sigma \psi=\pi$. Here $\pi$ is the natural projection of $\mathcal{S}$ onto $R / x_{0} \Re$. Both $\sigma$ and $\psi$ are homogeneous maps of degree zero.

The $d$ th homogeneous piece, $\psi(9)_{d}$, of $\psi(\mathscr{G})$ is obviously spanned by $\psi\left(F_{1}\right), \ldots, \psi\left(F_{n}\right)$. We claim that these elements are linearly independent over $k$. This argument is the same as the first paragraph of the proof of Theorem 1 in [3]. Hence we omit it. Set $V=\psi(9)_{d}$. Then $\operatorname{dim}_{k} V=n$.

Now $R$ is a graded ring. We shall denote the $j$ th homogeneous piece of $R$ by $R$. Since $\mathfrak{L}_{1}, \ldots, \mathfrak{L}_{s}$ are in generic $s$-position, we have $\operatorname{dim}_{k} \Re_{j}=\min \{s, \nu(j)\}$ for $j=0$, 1 .... In particular, we have the following dimensions:

$$
\operatorname{dim}_{k} \Re_{j}= \begin{cases}\nu(j) & \text { if } j=0,1, \ldots, d-1, \\ s & \text { if } j \geqslant d .\end{cases}
$$


Since $x_{0}$ is not a zero-divisor in $R$, multiplication by $x_{0}$ is a monomorphism. Thus, equation (4) implies $\Re / x_{0} \Re$ has the following form:

$$
\Re / x_{0} \Re=\bigoplus_{j=0}^{d}\left\{\Re_{j} / x_{0} \Re_{j-1}\right\} \text {. }
$$

In equation (5), the 0 th term is $k$. Now we note that the $j$ th homogeneous piece, $\tilde{\sigma}_{j}$, of $\mathcal{T}$ has dimension given by $\operatorname{dim}_{k} \mathcal{T}_{j}=\left(\begin{array}{c}n+j-1 \\ n-1\end{array}\right)$. But $\left(\begin{array}{c}n+j-1 \\ n-1\end{array}\right)=\operatorname{dim}_{k}\left\{\Re_{j} / x_{0} \Re_{j-1}\right\}$ for $j=0, \ldots, d-1$. Thus, $\sigma$ is an isomorphism in degrees $0,1, \ldots, d-1$. Consequently, $R / x_{0} \Re \cong \mathcal{T}_{0} \oplus \cdots \oplus \mathcal{T}_{d-1} \oplus\left\{\mathcal{T}_{d} / V\right\}$.

Now let

$$
\phi: \Re_{d-1} / x_{0} \Re_{d-2} \rightarrow \operatorname{Hom}_{k}\left(\frac{\Re_{1}}{x_{0} \Re_{0}}, \frac{\Re_{d}}{x_{0} \Re_{d-1}}\right)
$$

be the natural map induced by multiplication in $\Re / x_{0} \Re$. Let $m$ denote the homogeneous, maximal ideal in $R$. Then the annihilator of $m / x_{0} R$ in $R / x_{0} R$ is given by

$$
\operatorname{Ann}\left\{m / x_{0} \Re\right\}=\operatorname{ker} \phi \oplus\left\{\Re_{d} / x_{0} \Re_{d-1}\right\} .
$$

Since $x_{0}$ is not a zero-divisor in $R, t\left(\varrho_{1}, \ldots, E_{s}\right)=\operatorname{dim}_{k}\left\{\operatorname{Ann}\left\{m / x_{0} \Re\right\}\right\}$. Proofs of these last two facts can be found in [5].

Now equation (5) implies that $\left\{\Re_{d} / x_{0} \Re_{d-1}\right\} \cong T_{d} / V$. Since $\operatorname{dim}_{k}\left\{\widetilde{T}_{d} / V\right\}=$ $\left(\begin{array}{c}n+d-1 \\ n-1\end{array}\right)-n$, the theorem will follow from equation (6) once we have shown that $\phi$ is a monomorphism. So, we have reduced the proof of the theorem to showing that $\phi$ is injective when $\varepsilon_{1}, \ldots, E_{s}$ are in uniform position in $\mathbf{A}_{k}^{n+1}$.

Consider the following commutative diagram:

$$
\begin{array}{ccc}
\Re_{d-1} / x_{0} \Re_{d-2} & \stackrel{\phi}{\rightarrow} & \operatorname{Hom}_{k}\left(\frac{\Re_{1}}{x_{0} \Re_{0}}, \frac{\Re_{d}}{x_{0} \Re_{d-1}}\right) \\
\uparrow \sigma_{d-1} & \uparrow \sigma_{d}^{*} \\
\tilde{T}_{d-1} & \stackrel{\dot{\phi}}{\rightarrow} & \operatorname{Hom}_{k}\left(\widetilde{T}_{1}, \tau_{d}\right)
\end{array}
$$

In diagram (7), $\tilde{\phi}$ is the obvious monomorphism induced by multiplication in $T . \sigma_{d-1}$ is the $(d-1)$-piece of the epimorphism $\sigma$. We have previously noted that $\sigma_{d-1}$ is a $k$-vector space isomorphism. $\sigma_{d}^{*}$ is the surjection induced by the map $\sigma_{d}: T_{d} \rightarrow$ $\Re_{d} / x_{0} \Re_{d-1} \cong \mathcal{T}_{d} / V$.

Now let $w \in \operatorname{ker} \phi$. Since $\sigma_{d-1}$ is an isomorphism, there exists a $g \in T_{d-1}$ such that $\sigma_{d-1}(g)=w$. The commutativity of diagram (7) implies $\sigma_{d}^{*} \tilde{\phi}(g)=0$. Thus, $g \widetilde{T}_{1} \subseteq V$. In particular, there exist constants $\alpha_{i j} \in k$ such that $g Y_{i}=\sum_{j=1}^{n} \alpha_{i j} \psi\left(F_{j}\right)$, $i=1, \ldots, n$. Now suppose $g \neq 0$. Then $g Y_{1}, \ldots, g Y_{n}$ are linearly independent over $k$. Thus, the matrix $\left(\alpha_{i j}\right)$ is invertible. Let $\left(\beta_{i j}\right)$ be the inverse of $\left(\alpha_{i j}\right)$. Then $\psi\left(F_{i}\right)=$ $\sum_{j=1}^{n} \beta_{i j}\left(g Y_{j}\right), i=1, \ldots, n$. In particular, $\psi\left(F_{1}\right)$ and $\psi\left(F_{2}\right)$ are in the principal ideal $(g)$ in $\mathcal{T}$. Since $\left\{X_{0}, F_{1}, F_{2}\right\}$ is a regular sequence in $\mathcal{S},\left\{\psi\left(F_{1}\right), \psi\left(F_{2}\right)\right\}$ is a regular sequence in $\mathcal{T}$. But, this last fact implies the grade of $(g)$ is at least two, which is impossible. Therefore, $g=0$, and $\phi$ is a monomorphism. This completes the proof of the theorem. 


\section{REFERFNCES}

I. M. Baruch and W. C. Brown. A matrix computation for the Cohen-Macaulas type of s-lines in affine $(n+1)$-space. J. Algebra (to appear)

2. A. (jeramita and F. Orecchia. On the Cohen-Macaulal type of s-lines in $\mathbf{A}^{\prime \prime}$ '. J. Algebra 70 (1981), $116-140$

3. Mimimalh generating ideals defining certain tangent curves. J. Algebra 78 (1982), 36-57.

4. A. (ieramita and P. Maroscia. The ideal of forms tanishing at a finite set of points in $\mathbf{P}^{\prime \prime}$. Queen: Papers in Pure and Appl. Math., preprint.

5. L. (i. Roberts, A conjecture on (ohen-Macaulal type, C. R. Math. Rep. Acad. Sci. Canada 3 (1981). $43-48$.

Department of Mathematics, Michigan State University, East lansing, Michigan 48824 